\title{
The 6th Frankfurt Meeting for Surgery of Obesity and Metabolic Disorders
}

\author{
Rudolf A. Weiner Sylvia Weiner \\ Centre for Minimally Invasive Surgery, Section Bariatric Surgery, Hospital Sachsenhausen, Frankfurt/M., Germany
}

12 years after the first scientific meeting to the topic 'Surgery for Morbid Obesity' in Germany, the 5th Frankfurt meeting in 2008 extended the main topics surgery and obesity into 'Obesity and Metabolic Surgery'. Between 2002 and 2009, the meeting took place in the old town hall of Frankfurt, called Römer since centuries, which contributed to the special and familiar atmosphere. All lectures were presented in the plenary session room of the Parliament of Frankfurt am Main, but with the increasing interest in the field, the town hall became too small. Therefore, the 6th Frankfurt meeting changed the location from the town hall to the congress palace in 2010.540 experts in the field, more than initially expected, came and took part in the different sessions and workshops. The congress offered a comprehensive overview of the latest advances, breakthroughs and novelties in the minimally invasive treatment of this fatal modern age epidemic.

At the one end of the malnutrition scale, obesity is one of today's most blatantly visible - yet most neglected - public health problems. Paradoxically coexisting with malnutrition, an escalating global epidemic of overweight and obesity 'globesity' - spread over many parts of the world. If no immediate action takes place, millions will suffer from an considerable number of serious health disorders.

Obesity is a complex condition with serious social and psychological dimensions that affects virtually all age and socioeconomic groups and threatens to overwhelm both developed and developing countries. In 1995 there were an estimated 200 million obese adults worldwide and another 18 million children under the age of 5 that were classified as overweight. As of 2000 the number of obese adults has increased to over 300 million. Contrary to common expectations, the obesity epidemic is not restricted to industrialized societies. It is estimated that in developing countries over 115 million people suffer from obesity-related problems.

\section{KARGER}

Fax +497614520714

Information@Karger.de

www.karger.com
(C) 2011 S. Karger GmbH, Freiburg

$1662-4025 / 11 / 0047-0001 \$ 38.00 / 0$

Accessible online at:

www.karger.com/ofa
As commonly known, obesity surgery is the only effective strategy to treat severely obese patients. Obesity treatment should be individually tailored, and all crucial factors, such as sex, the degree of obesity, individual health risks as well as psychobehavioral and metabolic characteristics, should be taken into account.

The spectrum of the procedures in obesity surgery is subject to continuous change and improvement. During the last 2 years, laparoscopic sleeve gastrectomy (LSG) increasingly has attracted the attention of surgeons and patients. LSG is an effective first line operation to achieve definitive weight loss in suitable patients and is also applied as revision treatment if other surgical interventions did not succeed. Its advantages are that i) it is not as technically difficult as the laparoscopic biliopancreatic diversion duodenal switch, ii) it can lead to very good weight loss results in the medium term and iii) it can help to reverse co-morbidities, thereby further improving patient's health as well as lessening the risk if further surgical weight loss interventions should be necessary. LSG also has a strong short-term effect on the metabolic syndrome but, when compared with gastric bypass surgery, weight regain results in an earlier recurrence of the diabetes situation. Because LSG is a restrictive rather than a malabsorptive procedure, nutritional concerns are much less than with bypass surgeries. However, the quality control study in Germany showed that this type of surgery is accompanied by more major complications and a higher mortality than the gastric bypass intervention in experienced hands. The Achilles heel of LSG is still the staple line leak in the angle of His. Therefore, also another surgical weight loss intervention, namely gastroplication, attracts more and more attention. With this procedure the risk of leaks is nonexisting, and the procedure can be performed endoscopically. The mechanism of restriction seems to be comparable with that of sleeve gastrectomy. 
The new insights that are currently gained by studying surgical interventions for diabetes may be the most significant in the field of diabetes treatment since the discovery of insulin, opening the way to a completely new strategy of diabetes treatment: metabolic surgery. Metabolic surgery that bypasses the duodenum can normalize blood glucose levels within $24 \mathrm{~h}$ after surgery, bring type 2 diabetes in remission and minimize the needs for blood glucose-lowering medication. The reasons for these favorable developments are unclear, but there are some interesting hypotheses. The surgical treatment of diabetes raises hope of millions of diabetes patients. In Germany, more than 8 million patients suffer from diabetes mellitus type 2. Surgical diabetes treatment is a complex procedure and accompanied by considerable potential risks. On the other hand, drug treatment of diabetes mellitus type 2 is also associated with significant morbidity and mortality. Thus a considerable number of patients may be prompted to consider the apparently drastic measure of surgical intervention in the hope for cure even though the term 'cure' in should not be used in connection with type 2 diabetes is incorrect. If a person does not lose enough weight, regains weight or has natural progression of diabetes, blood glucose-lowering remedies may be needed, although possibly in smaller doses. The preferred term therefore is 'remission'. Because of the success of weight loss surgery to treat type 2 diabetes and transplant therapies to treat type 1 diabetes, a new American Diabetes Association consensus statement now defines partial, complete and prolonged remission categories. However, there still remains the question: 'Is diabetes mellitus type 2 a surgically treatable disease?' In the current supplemental issue of OBEsity FACTs, two articles of the group around Frenken demonstrate that surgical procedures like the duodenal switch which exclude the duodenum and the proximal jejunum from food passage have the potential to bring diabetes mellitus type 2 to remission. Remission means an euglycemic state with normal levels of serum insu- lin and glycosylated hemoglobin (HbA1c) while the patient must not follow any diet restrictions.

In the future, an evaluation of hormonal and genetic determinants of weight loss could also contribute to a better adjustment of individual therapy to a particular obese patient. Therefore, a multilevel obesity management network of mutually collaborating facilities should be established to provide individually tailored treatment. Centers of excellence in obesity management with multidisciplinary teams should provide comprehensive programs for the treatment of obesity based on evidence-based medicine. With this in mind, special courses for 'bariatric nurses 'and 'young surgeons' were organized during the Frankfurt meeting for the first time in Europe.

Last but not least, the Frankfurt Meeting was a successful test for the next great event, the 16th World Congress of the International Federation for the Surgery of Obesity and Metabolic Disorders (IFSO) which will be organized by the same team. The IFSO will come to Germany for the first time. During the period of abstract submission Germany changed the position from one of the last places during all IFSO congresses since 1996 to the top - 95 abstracts were submitted from German surgeons. All other countries traditionally active in the field of obesity surgery like Italy (58), France (25), Spain (34), USA (78) and Brazil (62) were behind for the first time. On the other hand, there are also contributions from countries such as India (36), showing high rates of malnutrition only two decades ago. These countries will increasingly contribute to the rise of 'globesity' over the next years.

On behalf of the International Federation for the Surgery of Obesity and Metabolic Disorders (IFSO) it is a great honor and pleasure for the German Society for the Surgery of Obesity to invite you to the XVI. Congress of the International Federation for the Surgery of Obesity and Metabolic Disorders (IFSO) in Hamburg. For more information look at $w w w . i f s o 2011 . d e$ and register for this event.
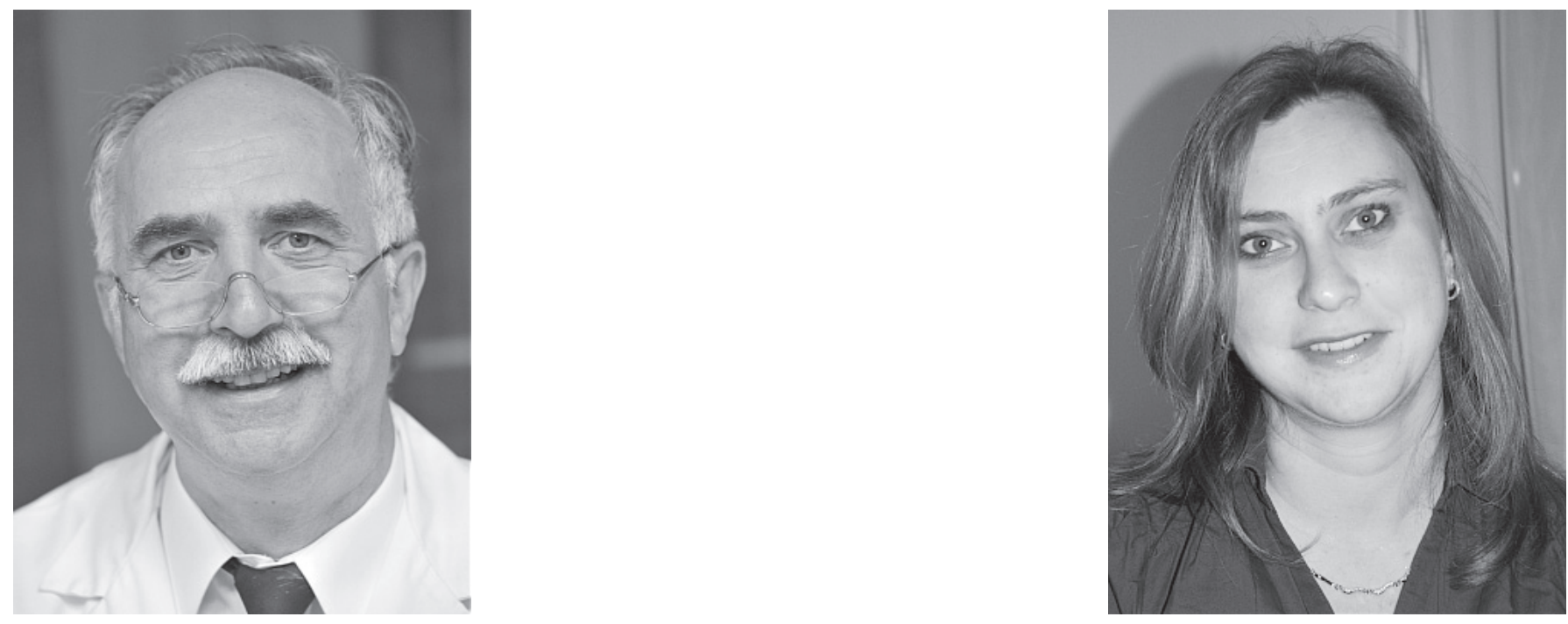